\title{
SPECTRAL GAPS OF DIRAC OPERATORS DESCRIBING GRAPHENE QUANTUM DOTS
}

\author{
RAFAEL D. BENGURIA, SØREN FOURNAIS, EDGARDO STOCKMEYER, \\ AND HANNE VAN DEN BOSCH
}

\begin{abstract}
The two-dimensional Dirac operator describes low-energy excitations in graphene. Different choices for the boundary conditions give rise to qualitative differences in the spectrum of the resulting operator. For a family of boundary conditions, we find a lower bound to the spectral gap around zero, proportional to $|\Omega|^{-1 / 2}$, where $\Omega \subset \mathbb{R}^{2}$ is the bounded region where the Dirac operator acts. This family contains the so-called infinite mass and armchair cases used in the physics literature for the description of graphene quantum dots.
\end{abstract}

\section{INTRODUCTION}

Graphene is a two-dimensional layer of carbon atoms forming a honeycomb lattice. Due to its hexagonal symmetry [24, 10, in absence of external fields, low-energy electronic excitations in an extended graphene sheet behave as Dirac fermions. Their dynamics is described effectively by the Hamiltonian

$$
H=\left(\begin{array}{cc}
T & 0 \\
0 & T
\end{array}\right) \quad \text { on } \quad \mathcal{H} \oplus \mathcal{H}
$$

where $\mathcal{H}=L^{2}\left(\mathbb{R}^{2}, \mathbb{C}^{2}\right)$ and $T$ is the massless two-dimensional Dirac operator

$$
T=v_{f} \hbar(-i \boldsymbol{\sigma} \cdot \nabla) \quad \text { on } \quad \mathcal{H},
$$

where $v_{f} \sim 10^{6} \mathrm{~m} / \mathrm{s}$ is the Fermi velocity. Here $\boldsymbol{\sigma}=\left(\sigma_{1}, \sigma_{2}\right)$ are the first two Pauli matrices. Through the orthogonal sum in (1), the operator $H$ takes into account contributions from the two inequivalent Dirac points (or valleys) $\boldsymbol{K}$ and $\boldsymbol{K}^{\prime}$ of the first Brillouin zone associated to the lattice. The components of a wavefunction in $\mathcal{H}$ describe the electronic density on each of the two triangular sublattices that constitute the honeycomb lattice. In many applications the contributions from the two valleys do not couple and the description is reduced to the study of the operator $T$ only (see 9 for a review).

When considering (quasi-)particles confined to some region $\Omega \subset \mathbb{R}^{2}$, one should impose boundary conditions that might break the block-diagonal structure of $H$. In the physics literature, much attention has been devoted to the so-called zigzag, armchair and infinite mass boundary conditions. The choice of a boundary condition influences the spectrum and therefore the transport properties of graphene ribbons and flakes, see for instance [1, 16, 25] for theoretical considerations or [20] for experimental observations. In particular, the presence of a gap in its spectrum allows to use a graphene device as a semiconductor.

We move on to a brief description of the above mentioned boundary conditions. Zigzag and armchair boundary conditions emerge from the tight-binding model and 
correspond to two different orientations of a straight lattice termination [1, 16]. The zigzag boundary conditions are known to be gapless, having zero as an eigenvalue of infinite multiplicity. From the mathematical point of view this has been observed in 21] (see also [12] for the absence of gaps of certain perturbed zigzag operators). The associated zero-energy states are well localized close to the boundary. In contrast, for armchair boundary conditions the presence of a gap has been noted (see e.g. [8, 17, 26]), and the lowest energy states are rather delocalized. Infinite mass boundary conditions, on the other hand, do not arise from the lattice termination. In fact, they were first studied in 1987 by Berry and Mondragon [6] for the operator $T$. They emerge from the Dirac operator with an effective mass term supported outside $\Omega$, as a limiting case when the mass tends to infinity, see [6, 22]. In the description of graphene, infinite mass boundary conditions have been also used to model quantum dots or nano-ribbons exhibiting a gap independent of the lattice orientation 1, 4, 9, 18. Zigzag and infinite mass boundary conditions do not couple the valleys and thus can be defined for $T$ too. Armchair boundary conditions do mix the valleys and make sense only when considering the full operator $H$.

In the present work, we obtain a lower bound on the gap size for $H$ with armchair or infinite mass boundary conditions in terms of $|\Omega|$. We start our analysis by studying the Dirac operator $T$ on a bounded simply connected domain $\Omega \subset \mathbb{R}^{2}$. In Theorem 1, we prove the desired estimate for a certain class of boundary conditions including the infinite mass case. The proof of the theorem will be given in Section2. In Section 3, through an elementary observation, we show that the lower bound for the infinite mass operator $T$ applies equally well to the operator $H$ with armchair boundary conditions. In this section, we also provide some further details on the physically relevant boundary conditions. We complete this introduction with the necessary definitions and the precise statement of the theorem. In our proof we follow the scheme developed by Bär in [3]. However in his case, Bär considers a manifold with curvature but without boundary. Towards the end of our proof we need to choose a trial function $f$. This is similar to what is done in [3]. In Bär's case his choice is dictated by the curvature of the manifold while in our case the choice is related to a boundary value problem which depends on our boundary conditions (see equation (4)).

Note added in proof: When preparing this manuscript, we were not aware of the work of Raulot [19] and we thank the referee for pointing out this reference to us. The case $n=2$ of [19, Theorem 1] is a generalization of our Theorem 1 with $B=1$, to arbitrary manifolds with boundary and so-called chiral boundary conditions. It is interesting to note that these chiral boundary conditions from differential geometry coincide for manifolds $\Omega \subset \mathbb{R}^{2}$ with the infinite mass boundary conditions known in the literature on graphene. Here, we stress the connection with the physics of graphene and provide a self-contained proof. It does not require sophisticated tools from differential geometry and also works in cases with limited regularity. Through an elementary observation (see Lemma 2 ) we can treat a family of boundary conditions $(0<B \leq 1)$.

1.1. Definitions and the main theorem. We consider a two-dimensional Dirac operator on a bounded domain $\Omega \subset \mathbb{R}^{2}$ with $C^{2}$-boundary $\partial \Omega$. Choosing appropriate units, the Dirac operator acts as the differential expression

$$
T \equiv-i \boldsymbol{\sigma} \cdot \nabla=\sigma_{1}\left(-i \partial_{1}\right)+\sigma_{2}\left(-i \partial_{2}\right) .
$$


Here, the Pauli matrices are defined as

$$
\sigma_{1}=\left(\begin{array}{cc}
0 & 1 \\
1 & 0
\end{array}\right), \quad \sigma_{2}=\left(\begin{array}{cc}
0 & -i \\
i & 0
\end{array}\right), \quad \sigma_{3}=\left(\begin{array}{cc}
1 & 0 \\
0 & -1
\end{array}\right),
$$

and we use the convention $\boldsymbol{\sigma} \cdot \boldsymbol{v}=\sum_{i=1}^{3} v_{i} \sigma_{i}$. We will write $D_{\eta}$ for the operator acting as $T$ on functions in the domain

$$
\mathcal{D}\left(D_{\eta}\right) \equiv\left\{u \in H^{1}\left(\Omega, \mathbb{C}^{2}\right) \mid P_{-, \eta} \gamma u=0\right\} .
$$

Here $\gamma$ is the trace operator on the boundary of $\Omega$ and the orthogonal projections $P_{ \pm, \eta}$ are defined as

$$
P_{ \pm, \eta}=1 / 2\left(1 \pm A_{\eta}\right), \quad A_{\eta}=\cos (\eta) \boldsymbol{\sigma} \cdot \boldsymbol{t}+\sin (\eta) \sigma_{3},
$$

where $\boldsymbol{t}$ is the unit vector tangent to the boundary. This is the only family of local boundary conditions making $T$ into a symmetric operator on $H^{1}(\Omega)$. A priori, $\eta$ can be any real function of $\partial \Omega$, but in the physically relevant cases it is a constant on each connected component of $\partial \Omega$. Infinite mass boundary conditions correspond to $\eta \equiv 0$ or $\eta \equiv \pi$. If $\eta$ is $C^{1}$ and $\cos \eta(s) \neq 0$ for all $s \in \partial \Omega, D_{\eta}$ is self-adjoint [5]. In this case, it follows from the compact embedding of $H^{1}(\Omega)$ in $L^{2}(\Omega)$ that the resolvent of $D_{\eta}$ is compact. Thus, its spectrum consists of eigenvalues of finite multiplicity accumulating only at $\pm \infty$.

For constant $\eta$ and simply connected domains, we obtain the following lower bound for the spectral gap.

Theorem 1. Take $\Omega \subset \mathbb{R}^{2}$ simply connected with $C^{2}$-boundary. Let $\eta$ be a constant such that $\cos \eta \neq 0$ and define $D_{\eta}$ as before. Define $B=\min (|\cos \eta /(1-\sin \eta)|, \mid(1-$ $\sin \eta) / \cos \eta) \mid$. If $\lambda$ is an eigenvalue of $D_{\eta}$, then

$$
\lambda^{2} \geq \frac{2 \pi}{|\Omega|} B^{2} .
$$

Remark 1. In physical units our lower bound for infinite mass boundary conditions $\left(B=1\right.$ in the theorem) gives a gap larger than $2 \sqrt{2 \pi} \hbar v_{f}|\Omega|^{-1 / 2}$. This means that in order to obtain a gap of $1 \mathrm{eV}$ one needs a domain with a diameter of about $10 \mathrm{~nm}$.

Remark 2. The bound is not sharp, but it is quite good, as a comparison with the case of a disc shows. When $B=1$, the lowest eigenvalue for a disc of unit radius is $k_{0}$, the smallest positive number such that $J_{0}\left(k_{0}\right)=J_{1}\left(k_{0}\right)$, where $J_{n}$ is the $n$-th Bessel function of the first kind (see [6]). Numerically, $k_{0} \approx 1.435$, and our lower bound reads

$$
k_{0}>\sqrt{2} \approx 1.414 .
$$

It is an open problem to obtain a sharp bound among all bounded two dimensional domains with the type of boundary conditions we consider.

Remark 3. The bound obtained by Raulot 19 is sharp and the case of equality is obtained by a manifold isomorphic to a half sphere $\mathbb{S}_{+}^{n}(r)$ with radius $r$, where $r$ depends on the first eigenvalue of the Dirac operator on the manifold with the boundary conditions he considers.

In the differential geometry literature, much attention has been devoted to lower bounds for the square of Dirac operators on surfaces. Most of these results deal with closed surfaces [13, 3]. For Dirac operators on two-dimensional manifolds with boundaries a less explicit bound has been derived in [15]. Our proof uses ideas from [3]. 
1.2. Notation. Before going further, we need to fix some notations. We will consider a fixed domain $\Omega$ with $C^{2}$-boundary $\partial \Omega$. We denote by $\boldsymbol{n}(s)$ and $\boldsymbol{t}(s)$ the outward normal and the tangent vector to the boundary at the point $s \in \partial \Omega$. The orientation of $\boldsymbol{t}$ is chosen such that $\boldsymbol{n}, \boldsymbol{t}$ is positively oriented, so we have $\boldsymbol{t} \cdot \nabla \boldsymbol{t}(s):=\partial_{s} \boldsymbol{t}(s)=-\kappa(s) \boldsymbol{n}(s)$, where $\kappa(s)$ is the curvature of the boundary. If $\boldsymbol{t}(s)=\left(t_{1}(s), t_{2}(s)\right)$, we define $t(s)=t_{1}(s)+i t_{2}(s)$, the tangent vector seen as a number in $\mathbb{C}$.

Functions in $H^{1}(\Omega)$ have well-defined traces on $\partial \Omega$, and since this will not cause confusion, we use the same notation for a function and its trace. In $L^{2}\left(\Omega, \mathbb{C}^{2}\right)$, the notations $\langle\cdot, \cdot\rangle$ and $\|\cdot\|$ will be used for the inner product and norm, respectively.

\section{ProOF OF THE THEOREM}

Before going into the proof of Theorem 1 , let us give a heuristic interpretation of the result. In [3] the following lower bound for the eigenvalues of the (classical) Dirac operator on closed surfaces $M$ of genus one (surfaces homeomorphic to a sphere) is proved:

$$
\lambda^{2} \geq \frac{4 \pi}{\operatorname{area}(M)} .
$$

The bound we obtain for an open and simply connected surface $\Omega$ with boundary condition $\eta \equiv 0, B=1$, is

$$
\lambda^{2} \geq \frac{2 \pi}{|\Omega|}
$$

At least formally, these particular boundary conditions provide the possibility to extend spinors in $\mathcal{D}(D)$ to the invertible double $\widetilde{\Omega}$, which is the closed surface obtained by glueing $\Omega$ to its mirror image. Details of this construction can be found in [7, chapter 9]. The bottom line is that an eigenspinor $u$ of $D$ can be extended to an eigenspinor $\widetilde{u}$ of the extended Dirac operator $\widetilde{D}$ by identifying $\widetilde{u} \approx(u,-u)$. Then, the bound of Theorem 1 follows from Bär's bound $(2)$, since area $(\widetilde{\Omega})=2|\Omega|$.

This argument can be made rigorous by considering closed surfaces $\widetilde{\Omega}_{\epsilon} \subset \mathbb{R}^{3}$ consisting of two copies of $\Omega$ in parallel planes with a distance $\epsilon$ between them, joined smoothly by a ribbon of width proportional to $\epsilon$. There is some work involved in computing explicitly the extension of $D$ to the curved ribbon and in checking that eigenspinors can be extended correspondingly. One has to make sure that the contribution of the curved part to the Rayleigh quotient tends to zero with $\epsilon$ in order to obtain the result. If $\Omega$ is not simply connected, we can still perform the doubling construction, but the resulting closed manifold will be homeomorphic to a torus or a surface of higher genus. In principle, this case can be treated using the results in 2 , that extend (2).

Instead of going through calculations with spinors on curved surfaces we will use the strategy from [3] taking care of the boundary terms. The boundary conditions for constant $\eta \notin\{0, \pi\}$ do not have the above described doubling property. However, one can extend the result for $\eta=0$ to the general case, as the following lemma shows.

Lemma 2. Take $D_{\eta}$ satisfying the hypotheses of Theorem 1, and

$$
B=\min (|\cos \eta /(1-\sin \eta)|,|(1-\sin \eta) / \cos \eta|) .
$$


If $\lambda_{\eta}$ is the eigenvalue of $D_{\eta}$ of smallest absolute value, then

$$
\lambda_{\eta}^{2} \geq B^{2} \lambda_{0}^{2}
$$

Proof. Assume $\eta \in(0, \pi / 2)$ such that $B=(1-\sin \eta) / \cos \eta \in(0,1)$. Take an eigenspinor $u$ of $D_{\eta}$ associated to the eigenvalue $\lambda_{\eta}$. Writing out the boundary conditions explicitly, we obtain $u_{2}=B t u_{1}$ on $\partial \Omega$. Then, we may write $u=v+w$, where $v=\left(\begin{array}{cc}B & 0 \\ 0 & 1\end{array}\right) u$. This gives $v \in \mathcal{D}\left(D_{0}\right)$, while $w \in \mathcal{D}\left(D_{\pi / 2}\right)$. Now we have

$$
\lambda_{\eta}^{2}\|u\|^{2}=\|T v+T w\|^{2}=\|T v\|^{2}+\|T w\|^{2}+2 \operatorname{Re}\langle T v, T w\rangle .
$$

The last two terms can be combined using the fact that $w$ has only its first component nonzero. We get

$$
\begin{aligned}
\|T w\|^{2}+2 & \operatorname{Re}\langle T v, T w\rangle \\
& =(1-B)^{2}\left\|\left(-i \partial_{1}-\partial_{2}\right) u_{1}\right\|^{2}+2 B(1-B)\left\|\left(-i \partial_{1}-\partial_{2}\right) u_{1}\right\|^{2} \\
& =\left(1-B^{2}\right)\left\|\left(-i \partial_{1}-\partial_{2}\right) u_{1}\right\|^{2} .
\end{aligned}
$$

Since $|B| \leq 1$ by definition, we have

$$
\lambda_{\eta}^{2}\|u\|^{2} \geq\left\|D_{0} v\right\|^{2} \geq \lambda_{0}^{2}\|v\|^{2} \geq \lambda_{0}^{2} B^{2}\|u\|^{2},
$$

which is the desired inequality. The other cases are analogous: it suffices to define $v=\left(\begin{array}{cc}-B & 0 \\ 0 & 1\end{array}\right) u$ when $\eta$ lies in $(\pi / 2, \pi)$ or $v=\left(\begin{array}{cc}1 & 0 \\ 0 & \pm B\end{array}\right) u$ for $\eta \in(\pi, 3 \pi / 2)$ and $\eta \in(3 \pi / 2,2 \pi)$.

Proof of Theorem 1. By the previous lemma we can restrict our attention to $\eta=0$, so to simplify notations, we will write $D_{0}=D$. Recall that the Pauli matrices satisfy the (anti-)commutation relations

$$
\left\{\sigma_{j}, \sigma_{k}\right\}=2 \delta_{j k}, \quad\left[\sigma_{j}, \sigma_{k}\right]=2 i \epsilon_{j k l} \sigma_{l}, \quad j, k, l \in\{1,2,3\},
$$

where $\delta_{j k}$ is the Kronecker delta and $\epsilon_{j k l}$ is the Levi-Civita symbol, which is totally antisymmetric and normalized by $\epsilon_{123}=1$. We start by a calculation for $C^{1}$-spinors $u, v \in \mathcal{D}(D)$

$$
\begin{aligned}
(D u, D v) & =\sum_{k, j} \int_{\Omega}\left(\partial_{k} u, \sigma_{k} \sigma_{j} \partial_{j} v\right)_{\mathbb{C}^{2}} \\
& =\sum_{k} \int_{\Omega}\left(\partial_{k} u, \partial_{k} v\right)_{\mathbb{C}^{2}}+i \sum_{k, j} \epsilon_{k j 3} \int_{\Omega}\left(\partial_{k} u, \sigma_{3} \partial_{j} v\right)_{\mathbb{C}^{2}} .
\end{aligned}
$$

In the second term we can integrate by parts using the antisymmetry of $\epsilon_{k j 3}$ and introduce the tangent vector at the boundary $\boldsymbol{t}=\left(-n_{2}, n_{1}\right)$. We obtain

$$
\begin{aligned}
\sum_{k, j} i \epsilon_{k j 3} \int_{\Omega}\left(\partial_{k} u, \sigma_{3} \partial_{j} v\right)_{\mathbb{C}^{2}} & =\sum_{k, j} i \epsilon_{k j 3} \int_{\Omega} \partial_{k}\left(u, \sigma_{3} \partial_{j} v\right)_{\mathbb{C}^{2}} \\
& =\sum_{k, j} i \epsilon_{k j 3} \int_{\partial \Omega} \boldsymbol{n}_{k}\left(u, \sigma_{3} \partial_{j} v\right)_{\mathbb{C}^{2}} \\
& =i \int_{\partial \Omega}\left(u, \sigma_{3} \boldsymbol{t} \cdot \nabla v\right)_{\mathbb{C}^{2}} .
\end{aligned}
$$

Since only the tangent derivative is involved, this term depends solely on the boundary values of $u$ and $v$. We can explicitly write out the spinor components and 
introduce the boundary condition in the form $u_{2}=t u_{1}$ :

$$
\left(u, \sigma_{3} \boldsymbol{t} \cdot \nabla v\right)_{\mathbb{C}^{2}}=u_{1}^{*} \boldsymbol{t} \cdot \nabla v_{1}-u_{2}^{*} \boldsymbol{t} \cdot \nabla v_{2}=-u_{1}^{*} v_{1} t^{*} t^{\prime}=-i u_{1}^{*} v_{1} \kappa(s)
$$

In the last equality, we used $\partial_{s} \boldsymbol{t}(s)=-\kappa(s) \boldsymbol{n}(s)$ (see Subsection 1.2). By density,

$$
(D u, D v)=(\nabla u, \nabla v)+\frac{1}{2} \int_{\partial \Omega}(u, v)_{\mathbb{C}^{2}}(s) \kappa(s) \mathrm{d} s
$$

holds for all $u, v \in \mathcal{D}(D)$.

For a real constant $\alpha$ and a real $C^{1}$-function $f$ we define a modified connection

$$
\widetilde{\partial}_{j}=\partial_{j}-i \alpha \sigma_{j}-\sigma_{f} \sigma_{j},
$$

where $\sigma_{f}:=\boldsymbol{\sigma} \cdot \nabla f$. For spinor fields $u, v$ we compute the product

$$
\begin{aligned}
\sum_{j}\left(\widetilde{\partial}_{j} u, \widetilde{\partial}_{j} v\right)_{\mathbb{C}^{2}}=\sum_{j} & \left(\left(\partial_{j} u, \partial_{j} v\right)_{\mathbb{C}^{2}}+\alpha^{2}\left(\sigma_{j} u, \sigma_{j} v\right)_{\mathbb{C}^{2}}+\left(\sigma_{f} \sigma_{j} u, \sigma_{f} \sigma_{j} v\right)_{\mathbb{C}^{2}}\right. \\
& -\left(\partial_{j} u,\left(i \alpha \sigma_{j}+\sigma_{f} \sigma_{j}\right) v\right)_{\mathbb{C}^{2}}-\left(\left(i \alpha \sigma_{j}+\sigma_{f} \sigma_{j}\right) u, \partial_{j} v\right)_{\mathbb{C}^{2}} \\
& \left.+i \alpha\left(\sigma_{j} u, \sigma_{f} \sigma_{j} v\right)_{\mathbb{C}^{2}}-i \alpha\left(\sigma_{f} \sigma_{j} u, \sigma_{j} v\right)_{\mathbb{C}^{2}}\right) \\
=( & \nabla u, \nabla v)_{\mathbb{C}^{2}}+\left(2 \alpha^{2}+2|\nabla f|^{2}\right)(u, v)_{\mathbb{C}^{2}} \\
& -\alpha\left((D u, v)_{\mathbb{C}^{2}}+(u, D v)_{\mathbb{C}^{2}}\right) \\
& -\sum_{j}\left(\left(\partial_{j} u, \sigma_{f} \sigma_{j} v\right)_{\mathbb{C}^{2}}+\left(\sigma_{f} \sigma_{j} u, \partial_{j} v\right)_{\mathbb{C}^{2}}\right) .
\end{aligned}
$$

By the anti-commutation relations, we obtain

$$
\sum_{j}\left(\sigma_{f} \sigma_{j} u, \partial_{j} v\right)_{\mathbb{C}^{2}}=-i\left(u, \sigma_{f} D v\right)_{\mathbb{C}^{2}}+2 \sum_{j}\left(\partial_{j} f\right)\left(u, \partial_{j} v\right)_{\mathbb{C}^{2}},
$$

$\mathrm{SO}$

$$
\begin{aligned}
\sum_{j}\left(\widetilde{\partial}_{j} u, \widetilde{\partial}_{j} v\right)_{\mathbb{C}^{2}}=( & \nabla u, \nabla v)_{\mathbb{C}^{2}}+\left(2 \alpha^{2}+2|\nabla f|^{2}\right)(u, v)_{\mathbb{C}^{2}} \\
& -\alpha\left((D u, v)_{\mathbb{C}^{2}}+(u, D v)_{\mathbb{C}^{2}}\right) \\
& -\sum_{j}\left(\left(\partial_{j} u, \sigma_{f} \sigma_{j} v\right)_{\mathbb{C}^{2}}+2\left(\partial_{j} f\right)\left(u, \partial_{j} v\right)_{\mathbb{C}^{2}}\right)+i\left(u, \sigma_{f} D v\right)_{\mathbb{C}^{2}} .
\end{aligned}
$$

We are interested in the integral over $\Omega$ of the above quantity with the weight $e^{-2 f}$. If $u, v \in \mathcal{D}\left(D^{2}\right)$, then we obtain using (3)

$$
\begin{aligned}
\left\langle e^{-2 f} u, D^{2} v\right\rangle & =\left\langle D e^{-2 f} u, D v\right\rangle \\
& =\left\langle\nabla e^{-2 f} u, \nabla v\right\rangle+\int_{\partial \Omega} e^{-2 f} \frac{\kappa}{2}(u, v)_{\mathbb{C}^{2}} \\
& =\int_{\Omega} e^{-2 f}\left((\nabla u, \nabla v)_{\mathbb{C}^{2}}-2 \sum_{j}\left(\partial_{j} f\right)\left(u, \partial_{j} v\right)_{\mathbb{C}^{2}}\right)+\int_{\partial \Omega} e^{-2 f} \frac{\kappa}{2}(u, v)_{\mathbb{C}^{2}} .
\end{aligned}
$$

And by integration by parts

$$
\begin{aligned}
\sum_{j} \int_{\Omega} e^{-2 f}\left(\partial_{j} u, \sigma_{f} \sigma_{j} v\right)_{\mathbb{C}^{2}}= & -i\left\langle e^{-2 f} u, \sigma_{f} D v\right\rangle+\left\langle\left(-\Delta f+2|\nabla f|^{2}\right) e^{-2 f} u, v\right\rangle \\
& +\int_{\partial \Omega} e^{-2 f}\left(u, \sigma_{f} \boldsymbol{\sigma} \cdot \boldsymbol{n} v\right)_{\mathbb{C}^{2}} .
\end{aligned}
$$


Inserting these identities, we obtain

$$
\begin{aligned}
\sum_{j} \int_{\Omega} e^{-2 f} & \left(\widetilde{\partial}_{j} u, \widetilde{\partial}_{j} v\right)_{\mathbb{C}^{2}}=\left\langle e^{-2 f} u, D^{2} v\right\rangle+2 \sum_{j}\left\langle e^{-2 f} u,\left(\partial_{j} f\right) \partial_{j} v\right\rangle \\
& +\left\langle e^{-2 f}\left(2 \alpha^{2}+2|\nabla f|^{2}\right) u, v\right\rangle-\alpha\left\langle e^{-2 f} u, D v\right\rangle-\alpha\left\langle e^{-2 f} D u, v\right\rangle \\
& +2 i\left\langle e^{-2 f} u, \sigma_{f} D v\right\rangle+\left\langle e^{-2 f} u,\left(\Delta f-2|\nabla f|^{2}\right) v\right\rangle \\
& -2 \sum_{j}\left\langle e^{-2 f} u,\left(\partial_{j} f\right) \partial_{j} v\right\rangle \\
& +\int_{\partial \Omega} e^{-2 f}\left(-\frac{\kappa}{2}(u, v)_{\mathbb{C}^{2}}-\left(u, \sigma_{f} \boldsymbol{\sigma} \cdot \boldsymbol{n} v\right)_{\mathbb{C}^{2}}\right) .
\end{aligned}
$$

Note that the four terms containing first derivatives of $f$ cancel exactly. Applying this identity with $v=u$ and $D u=\lambda u$, it reduces to

$$
\begin{aligned}
\sum_{j} \int_{\Omega} e^{-2 f}\left(\widetilde{\partial}_{j} u, \widetilde{\partial}_{j} u\right)_{\mathbb{C}^{2}}= & \left.\lambda^{2}+2 \alpha^{2}-2 \alpha \lambda\right)\left\|e^{-f} u\right\|^{2} \\
& +2 i \lambda\left\langle u, \sigma_{f} u\right\rangle+\left\langle e^{-2 f} u, \Delta f u\right\rangle \\
& +\int_{\partial \Omega} e^{-2 f}\left(-\frac{\kappa}{2}(u, u)_{\mathbb{C}^{2}}-\left(u, \sigma_{f} \boldsymbol{\sigma} \cdot \boldsymbol{n} u\right)_{\mathbb{C}^{2}}\right) .
\end{aligned}
$$

Now we use the fact that the left hand side is real and nonnegative, and choose $\alpha=\lambda / 2$ in order to minimize the coefficient of the first term. We obtain the inequality

$$
0 \leq \frac{\lambda^{2}}{2}\left\|e^{-f} u\right\|^{2}+\left\langle e^{-2 f} u,(\Delta f) u\right\rangle+\int_{\partial \Omega} e^{-2 f}\left(-\frac{\kappa}{2}(u, u)_{\mathbb{C}^{2}}-\operatorname{Re}\left(u, \sigma_{f} \boldsymbol{\sigma} \cdot \boldsymbol{n} u\right)_{\mathbb{C}^{2}}\right) .
$$

Using anti-commutation relations, $\operatorname{Re}\left(u, \sigma_{f} \boldsymbol{\sigma} \cdot \boldsymbol{n} u\right)_{\mathbb{C}^{2}}=(\boldsymbol{n} \cdot \nabla f)(u, u)_{\mathbb{C}^{2}}$, so we obtain

$$
\frac{\lambda^{2}}{2}\left\|e^{-f} u\right\|^{2} \geq-\left\langle e^{-2 f} u,(\Delta f) u\right\rangle+\int_{\partial \Omega} e^{-2 f}(u, u)_{\mathbb{C}^{2}}\left(\frac{\kappa}{2}+\boldsymbol{n} \cdot \nabla f\right) .
$$

This suggests to take $f$ solving, for some $C \in \mathbb{R}$,

$$
\begin{cases}\Delta f=C & \text { in } \Omega, \\ \boldsymbol{n} \cdot \nabla f=-\kappa / 2 & \text { in } \partial \Omega .\end{cases}
$$

To see that such an $f$ exists, set $f_{0}(x)=C|x|^{2} / 4$. By [11, Theorem 3.40, p138], we can find $f_{h}$ satisfying

$$
\begin{cases}\Delta f_{h}=0, & \text { in } \Omega, \\ \boldsymbol{n} \cdot \nabla f_{h}=-\kappa / 2-\boldsymbol{n} \cdot \nabla f_{0}, & \text { in } \partial \Omega,\end{cases}
$$

provided $\int_{\partial \Omega}\left(-\kappa / 2-\boldsymbol{n} \cdot \nabla f_{0}\right)=0$. Since $\Omega$ is simply connected, $\int_{\partial \Omega} \kappa=2 \pi$. On the other hand, $\int_{\partial \Omega} \boldsymbol{n} \cdot \nabla f_{0}=\int_{\Omega} \Delta f_{0}=C|\Omega|$. So with the choice $C=-\pi /|\Omega|$, $f_{h}+f_{0}$ satisfies (4). The final result is

$$
\lambda^{2} \geq-2 C=\frac{2 \pi}{|\Omega|} .
$$




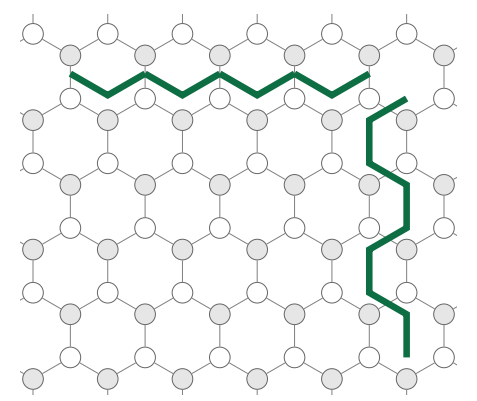

Figure 1. A honeycomb lattice, where the gray and white dots represent carbon atoms on each of the two triangular sublattices. The thick lines indicate the zigzag boundary (above) and armchair boundary (right).

\section{Application to The tWo-VALley Description of GRAPHENE}

We now apply our results to the description of electronic excitations in graphene as a four-component spinor with

$$
H=\left(\begin{array}{cc}
T & 0 \\
0 & T
\end{array}\right)
$$

If the boundary conditions are local and uniform, the four-spinors should fulfill

$$
P_{-}(A) \psi:=\frac{1}{2}(1-A) \psi=0 \quad \text { on } \partial \Omega .
$$

Here $A$ is a unitary matrix that belongs to a four-parameter family, see [1 for its explicit form. For simplicity we will restrict our attention to the boundary conditions most commonly used in the physics literature, following the notations of [1].

Zigzag boundary conditions. Zigzag boundary conditions arise from the tightbinding model when the honeycomb lattice is terminated in a direction perpendicular to the bonds, see Figure 1. In this case,

$$
A=\left(\begin{array}{cc}
\sigma_{3} & 0 \\
0 & -\sigma_{3}
\end{array}\right) .
$$

Thus, these boundary conditions do not mix the two valleys. We obtain two copies of $D_{\pi / 2}$, which is not self-adjoint on $H^{1}\left(\Omega, \mathbb{C}^{2}\right)$ and has zero as an eigenvalue of infinite multiplicity [21].

Infinite Mass boundary conditions. These boundary conditions have been used as an effective model describing a graphene quantum dot or nanoribbon, when a detailed microscopical description of the boundary is lacking [4, 9, 18]. In addition, infinite mass boundary conditions are obtained as a limiting case of $T$ acting on $L^{2}\left(\mathbb{R}^{2}\right)$ with a mass term $M(x)=M\left(1-\chi_{\Omega}(x)\right)$ when the mass tends to infinity. Physically, such a term is the continuum limit of a staggered potential (opposite signs on both sublattices) and several mechanisms to realize this in practice have been proposed [14, 23, 27].

The matrix giving the boundary conditions here is

$$
A=\left(\begin{array}{cc}
\sigma \cdot t & 0 \\
0 & -\sigma \cdot t
\end{array}\right) .
$$


This does not mix the valleys and gives a block diagonal operator with $D_{0}$ and $D_{\pi}$ on the diagonal. Therefore, the operator is self-adjoint with domain $\mathcal{D}\left(D_{0}\right) \oplus \mathcal{D}\left(D_{\pi}\right) \subset$ $H^{1}\left(\Omega, \mathbb{C}^{4}\right)$ and the estimate of Theorem 1 holds.

Armchair boundary conditions. Armchair boundary conditions also arise from the termination of a lattice, when the direction of the boundary is parallel to the bonds (see Figure 1). It has been noted in some particular cases that these boundary conditions give rise to a gap in the spectrum around zero (see for instance [8, 17, 26]). The boundary conditions are determined by

$$
A=\left(\begin{array}{cc}
0 & \nu^{*} \boldsymbol{\sigma} \cdot \boldsymbol{t} \\
\nu \boldsymbol{\sigma} \cdot \boldsymbol{t} & 0
\end{array}\right)
$$

where $|\nu|=1$. We show how these boundary conditions can be brought into a blockdiagonal form in order to apply our theorem. Using the unitary transformation

$$
U_{\nu}=\left(\begin{array}{cc}
\nu 1_{\mathbb{C}^{2}} & 0 \\
0 & 1_{\mathbb{C}^{2}}
\end{array}\right)
$$

we can restrict our attention to the case $\nu=1$. Consider the unitary transformation

$$
U_{p}=\left(\begin{array}{cccc}
1 & 0 & 0 & 0 \\
0 & 0 & 0 & 1 \\
0 & 0 & 1 & 0 \\
0 & 1 & 0 & 0
\end{array}\right)
$$

corresponding to a permutation of the second and fourth spinor components. This transforms the boundary conditions in

$$
\widetilde{A}=U_{p} A U_{p}^{*}=\left(\begin{array}{cc}
\boldsymbol{\sigma} \cdot \boldsymbol{t} & 0 \\
0 & \boldsymbol{\sigma} \cdot \boldsymbol{t}
\end{array}\right),
$$

and the Hamiltonian as

$$
\widetilde{H}=U_{p} H U_{p}^{*}=\left(\begin{array}{cc}
0 & T \\
T & 0
\end{array}\right) .
$$

After this transformation, a four-spinor $\psi$ in the domain of $\widetilde{H}$ can be written as $\psi=\left(\begin{array}{l}u_{1} \\ u_{2}\end{array}\right)$ with $u_{1}, u_{2} \in \mathcal{D}\left(D_{0}\right)$. A short calculation shows that the same holds for the adjoint: $\phi \in \mathcal{D}\left(\widetilde{H}^{*}\right)$ if and only if $\phi=\left(\begin{array}{l}v_{1} \\ v_{2}\end{array}\right)$ with $v_{1}, v_{2} \in \mathcal{D}\left(D_{0}^{*}\right)=\mathcal{D}\left(D_{0}\right)$. Thus, $H$ is self-adjoint on a domain included in $H^{1}\left(\Omega, \mathbb{C}^{4}\right)$. Furthermore,

$$
\|\widetilde{H} \psi\|^{2}=\left\|\left(\begin{array}{c}
D_{0} u_{2} \\
D_{0} u_{1}
\end{array}\right)\right\|^{2} \geq \frac{2 \pi}{|\Omega|}\|\psi\|^{2} .
$$

In other words, the estimate of Theorem 1 holds in this case as well.

Acknowledgments. This work has been supported by the Iniciativa Científica Milenio (Chile) through the Millenium Nucleus RC-120002 "Física Matemática" . R.B. has been supported by Fondecyt (Chile) Projects \# 112-0836 and \#114-1155. S.F. acknowledges partial support from a Sapere Aude grant from the Danish Councils for Independent Research, Grant number DFF-4181-00221. E.S has been partially funded by Fondecyt (Chile) project \# 114-1008. H. VDB. acknowledges support from Conicyt (Chile) through CONICYT-PCHA/Doctorado Nacional/2014. This work was carried out while S.F. was invited professor at Pontificia Universidad Católica de Chile. 


\section{REFERENCES}

[1] A. R. Akhmerov and C. W. J. Beenakker, Boundary conditions for Dirac fermions on a terminated honeycomb lattice, Phys. Rev. B 77 (2008), 085423.

[2] B. Ammann and C. Bär, Dirac eigenvalue estimates on surfaces, Math. Z. 240 (2002), no. 2, 423-449.

[3] C. Bär, Lower eigenvalue estimates for Dirac operators, Math. Ann. 293 (1992), no. 1, 39-46.

[4] C. G. Beneventano, I. Fialkovsky, E. M. Santangelo, and D. V. Vassilevich, Charge density and conductivity of disordered berry-mondragon graphene nanoribbons, The European Physical Journal B 87 (2014), no. 3, 1-9.

[5] R. Benguria, S. Fournais, E. Stockmeyer, and H. Van Den Bosch, Self-adjointness of TwoDimensional Dirac operators in Domains, Annales Herin Poincaré (online first), DOI 10.1007/s00023-017-0554-5.

[6] M. V. Berry and R. J. Mondragon, Neutrino billiards: time-reversal symmetry-breaking without magnetic fields, Proc. Roy. Soc. London Ser. A 412 (1987), no. 1842, 53-74.

[7] B. Booß-Bavnbek and K. P. Wojciechowski, Elliptic boundary problems for Dirac operators, Mathematics: Theory \& Applications, Birkhäuser Boston, Inc., Boston, MA, 1993.

[8] L. Brey and H. A. Fertig, Electronic states of graphene nanoribbons studied with the dirac equation, Phys. Rev. B 73 (2006), 235411.

[9] A. H. Castro Neto, F. Guinea, N. M. R. Peres, K. S. Novoselov, and A. K. Geim, The electronic properties of graphene, Rev. Mod. Phys. 81 (2009), 109-162.

[10] C.L. Fefferman and M.I. Weinstein, Honeycomb lattice potentials and Dirac points, J. Amer. Math. Soc. 25 (2012), no. 4, 1169-1220.

[11] G. B. Folland, Introduction to partial differential equations, second ed., Princeton University Press, Princeton, NJ, 1995.

[12] P. Freitas and P. Siegl, Spectra of graphene nanoribbons with armchair and zigzag boundary conditions, Rev. Math. Phys. 26 (2014), no. 10, 1450018, 32.

[13] Th. Friedrich, Der erste Eigenwert des Dirac-Operators einer kompakten, Riemannschen Mannigfaltigkeit nichtnegativer Skalarkrümmung, Math. Nachr. 97 (1980), 117-146.

[14] G. Giovannetti, P. A. Khomyakov, G. Brocks, P. J. Kelly, and J. van den Brink, Substrateinduced band gap in graphene on hexagonal boron nitride: Ab initio density functional calculations, Phys. Rev. B 76 (2007), 073103.

[15] O. Hijazi, S. Montiel, and X. Zhang, Eigenvalues of the Dirac operator on manifolds with boundary, Comm. Math. Phys. 221 (2001), no. 2, 255-265.

[16] E. McCann and V. I. Fal'ko, Symmetry of boundary conditions of the dirac equation for electrons in carbon nanotubes, Journal of Physics: Condensed Matter 16 (2004), no. 13, 2371.

[17] A. Orlof, J. Ruseckas, and I. V. Zozoulenko, Effect of zigzag and armchair edges on the electronic transport in single-layer and bilayer graphene nanoribbons with defects, Phys. Rev. B 88 (2013), 125409.

[18] L. A. Ponomarenko, F. Schedin, M. I. Katsnelson, R. Yang, E. W. Hill, K. S. Novoselov, and A. K. Geim, Chaotic Dirac billiard in graphene quantum dots, Science 320 (2008), no. 5874, 356-358.

[19] S. Raulot, The Hijazi inequality on manifolds with boundary, J. Geom. Phys. 56 (2006), 2189-2202.

[20] K. A. Ritter and Joseph W. Lyding, The influence of edge structure on the electronic properties of graphene quantum dots and nanoribbons, Nat. Mater. (2009), 235.

[21] K. M. Schmidt, A remark on boundary value problems for the Dirac operator, Quart. J. Math. Oxford Ser. (2) 46 (1995), no. 184, 509-516.

[22] E. Stockmeyer and S. Vugalter, Infinite mass boundary conditions for Dirac operators, Preprint (2016), arXiv:1603.09657

[23] D. Subramaniam, F. Libisch, Y. Li, C. Pauly, V. Geringer, R. Reiter, T. Mashoff, M. Liebmann, J. Burgdörfer, C. Busse, T. Michely, R. Mazzarello, M. Pratzer, and M. Morgenstern, Wave-function mapping of graphene quantum dots with soft confinement, Phys. Rev. Lett. 108 (2012), 046801.

[24] P.R. Wallace, The band theory of graphite, Phys. Rev. 71 (1947), no. 9, 622. 
[25] J. Wurm, A. Rycerz, İ ç Adagideli, M. Wimmer, K. Richter, and H. U. Baranger, Symmetry classes in graphene quantum dots: Universal spectral statistics, weak localization, and conductance fluctuations, Phys. Rev. Lett. 102 (2009), 056806.

[26] H. Zheng, Z. F. Wang, T. Luo, Q. W. Shi, and J. Chen, Analytical study of electronic structure in armchair graphene nanoribbons, Phys. Rev. B 75 (2007), 165414.

[27] S. Y. Zhou, G.-H. Gweon, A. V. Fedorov, P. N. First, W. A. de Heer, D.-H. Lee, F. Guinea, A. H. Castro Neto, and A. Lanzara, Substrate-induced bandgap opening in epitaxial graphene, Nat. Mater. (2007), 770.

Rafael D. Benguria, Edgardo Stockmeyer and Hanne Van Den Bosch, Instituto de Física, Pontificia Universidad Católica de Chile, Vicuña Mackenna 4860, Santiago 7820436, Chile.

Søren Fournais, Department of Mathematics, Aarhus University, Ny Munkegade 118, DK-8000 Aarhus, Denmark 